\title{
Eva Pebay-Peroula : un parcours original, de la physique atomique à la biologie structurale
}

Interview réalisée par Claire Schlenker, de la commission Femmes et Physique (F\&P) de la Société Française de Physique, le 4 mai 2016, dans les nouveaux locaux spacieux de I'Institut de Biologie Structurale (IBS), sur le campus EPN (European Photons and Neutrons) du Polygone scientifique de Grenoble. Ce campus regroupe I'ILL (Institut Laue-Langevin, source de neutrons), I'ESRF (source européenne de rayonnement synchrotron), ainsi qu'un laboratoire de l'EMBL (European Molecular Biology Laboratory).

F\&P : Eva, tu as 59 ans, tu es professeure à l'Université Joseph Fourier (Université Grenoble Alpes), spécialiste de biologie structurale. Ta brillante contribution à la compréhension des fonctions des protéines membranaires t’a conduite à l'Académie des sciences. Peux-tu nous expliquer comment est née ta vocation scientifique?

Eva Pebay-Peroula (EPP) : Mon parcours n'a pas été direct. J'étais très intéressée par les sciences dès mes études secondaires au lycée Stendhal de Grenoble. Je me suis donc orientée vers les classes préparatoires aux grandes écoles. Passionnée par la météorologie, je suis entrée dans une école de météo. Déçue par ce domaine, j'ai démissionné au bout d'une année. Je me suis réorientée vers des études de physique à la Faculté des Sciences de Grenoble, en vue d'une carrière dans l'enseignement. Après une maitrise de physique, j'ai été reçue à l'agrégation de sciences physiques. J'ai ensuite enseigné deux ans dans l'enseignement secondaire. Lors de mon congé d'une année pour la naissance de mon premier enfant, j'ai entrepris la préparation d'un DEA de physique, matière et rayonnement (Master 2). Ce n'est que le jour de ma soutenance de stage de DEA que, frappée par l'intérêt du jury pour mes travaux, un déclic s'est produit, et j'ai alors décidé de m'orienter vers la recherche. J'ai ensuite préparé une thèse en physique atomique sous la direction de Rémy Jost, au sein du Service national des champs intenses (maintenant le laboratoire LNCMI).
F\&P : Quelle est l'origine de ton orientation vers la biologie structurale?

EPP : Comme il y avait alors très peu de postes en physique atomique, j'ai d'abord été détachée de l'enseignement secondaire au CNRS pendant une année. J'ai ensuite obtenu pendant cinq ans un poste de scientifique à l'ILL, alors que je n'avais aucune compétence en diffusion neutronique. Je me suis orientée vers la biophysique, guidée par Jo Zaccai, puis introduite à la biologie structurale par Mogens Lehmann et Claudine Cohen-Addad. Après deux années, j’ai pu obtenir en 1989 un poste de Maître de conférences en physique/biologie structurale à l'Université Joseph Fourier (UJF), tout en poursuivant mes recherches à l'ILL. J'ai rejoint l'Institut de Biologie Structurale qui a été créé en 1992. L'atmosphère qui régnait à l'ILL dans les années 1989-92 était très stimulante, en particulier sous l'impulsion de Bernard Jacrot qui a beaucoup œuvré pour l'interface physique-biologie et la biologie structurale, et grâce au rôle moteur de Dick Wade.

F\&P : Comment s'est déroulée ensuite ta carrière ?

EPP : J'ai pu développer dans les années 90 deux nouveaux sujets, sans beaucoup de contrainte, car nous étions alors des pionniers. Mon sujet principal était l'étude des protéines membranaires, sujet que j'ai mené en collaboration avec J.P. Rosenbusch de l'Université de Bâle, en commençant avec des porines bactériennes. J'ai aussi abordé le problème des ribosomes, en collaboration avec

\section{Parcours}

- 1979 Agrégation de sciences physiques

-1979-1981 Professeure de lycée

- 1986 Thèse de doctorat en physique atomique, soutenue à I'UJF

-1986-1987 Détachement au CNRS, Service national des champs intenses, Grenoble

-1987-1992 Scientifique à I'Institut Laue-Langevin

Eva Pebay-Peyroula est née en 1956.

\section{Distinctions}

- 2004 Membre de I'Académie des sciences

- 2005 Médaille d'argent du CNRS

- 2016 Officier de la Légion d'honneur
- 1989-1995 Maître de conférences, puis, depuis 1995, Professeure à I'UJF

- 2004-2014 Directrice de I'Institut de Biologie Structurale (IBS)

- Depuis 2006 Membre de I'Institut Universitaire de France (IUF) senior

- 2010-2012 Présidente du Conseil d'administration de l'Agence nationale de la recherche (ANR)

eva.pebay-peyroula@ibs.fr 
1. Illustration d'un mécanisme cellulaire : le transporteur $A A C$ permet aux nucléotides ATP et ADP de traverser la membrane qui protège la mitochondrie, un des compartiments internes de la cellule.

(a) Le cristal de $A A C$, d'environ $40 \times 20 \times 5 \mu m$, maintenu dans une boucle de nylon, est placé dans le faisceau de rayons $X$ sous un flux d'azote refroidi à $100 \mathrm{~K}$, pour limiter les dégâts d'irradiation pendant l'enregistrement des données de diffraction.

(b) La carte de densité électronique expérimentale (en blanc), obtenue grâce au rayonnement synchrotron de l'ESRF, a permis de tracer les hélices formées par l'enchaînement des acides aminés de AAC (en rouge), dévoilant ainsi le squelette d'une grande partie de la protéine.

(c) Schéma d'une molécule d'ADP, à la même échelle que la molécule AAC en (d).

(d) Structure atomique de AAC, obtenue avec une résolution de 2,2 $\AA$. AAC comporte 293 acides aminés et plus de 4000 atomes. La surface de la protéine est représentée en cyan et laisse montrer par transparence son squelette. La structure a révélé une cavité ouverte vers l'extérieur (en haut sur la figure) prête à recevoir une molécule d’ADP (représentée en $(c)$ ) et permettre son passage de l'autre côté de la membrane (schématisée par le rectangle beige). La flèche bleue montre comment
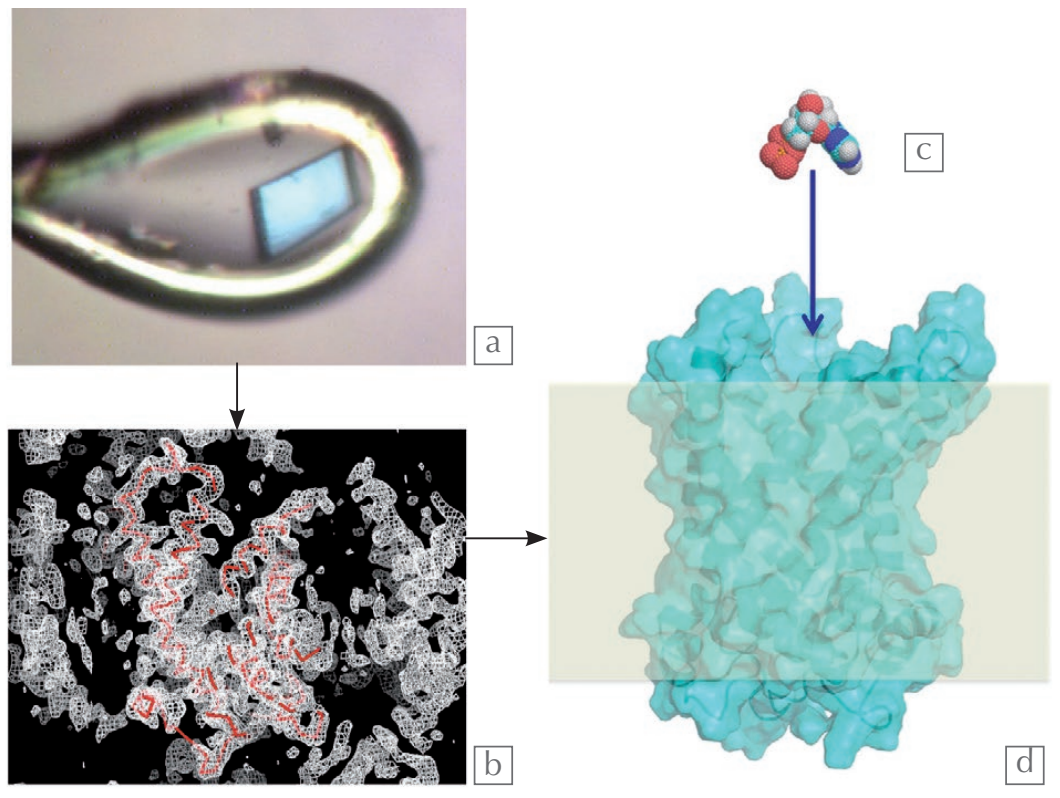
le nucléotide va entrer dans la cavité du transporteur.

Ada Jonath (Prix Nobel de chimie en 2009). En 1995, j’ai obtenu un poste de professeure à l'UJF et ai été de 1994 à 1999 membre junior de l'Institut Universitaire de France. J'ai poursuivi l'étude des membranes et ai pu créer à l'IBS une jeune équipe universitaire, grâce au soutien de Daniel Bloch et Claude Feuerstein, alors présidents de l'UJF. En 1995, une nouvelle approche de cristallisation de protéines membranaires a été inventée par l'équipe de J. Rosenbusch. Dans les années 19952000, j'ai collaboré avec cette équipe pour participer au développement de cette méthode. J'ai étudié, toujours en collaboration avec J. Rosenbusch, des rhodopsines bactériennes, protéines qui ont la capacité d'absorber la lumière visible et d'utiliser cette énergie pour des processus cellulaires importants. L'une d'elles réalise, grâce à cette énergie, la synthèse de l'adénosine triphosphate (ATP), ce qui constitue le premier maillon de la conversion de l'énergie lumineuse en énergie chimique utilisable par la bactérie.

F\&P : Quelles sont tes activités de recherche plus récentes? EPP : J'ai étudié ensuite un transporteur mitochondrial, appelé AAC, qui permet d'exporter l'ATP synthétisé dans la mitochondrie. La molécule d'ATP s'hydrolyse en ADP en libérant de l'énergie. La plupart des réactions enzymatiques réalisées dans la cellule utilisent cette énergie. La mitochondrie, compartiment cellulaire dans lequel s'effectue la régénération d'ADP en ATP, est donc une véritable usine énergétique de la cellule. Les résultats obtenus, notamment grâce aux études à l'ESRF, étaient les premiers sur cette famille de transporteurs. La résolution de la structure, publiée en 2003, avait bénéficié d'une excellente collaboration avec G. Brandolin, biochimiste au CEA Grenoble. La structure a permis de comprendre comment l'ADP peut entrer dans une énorme cavité (fig. 1). Grâce à la collaboration avec l'équipe de C. Chipot, chimiste théoricien de Nancy, nous avons pu comprendre que l'ADP, molécule portant trois charges négatives, est attiré grâce au potentiel électrostatique créé par le transporteur AAC. Nous avons aussi pu expliquer les causes moléculaires de dysfonctionnement dû à des mutations de ce transporteur, identifiées chez des personnes atteintes de certaines formes de myopathies. Avec mon équipe, je poursuis ces études sur d'autres types de transporteurs.

F\&P : Quelles responsabilités as-tu eues au cours de ta carrière? EPP : J'ai dirigé mon équipe de recherche depuis les années 1990, avec d'abord trois à quatre personnes et aujourd'hui une dizaine. J'ai été directrice adjointe de l'IBS de 2002 à 2004, puis directrice de 2004 à 2014. J'ai aussi été présidente du Conseil d'administration de l'Agence nationale de la recherche (ANR), de 2010 à 2012.

$\mathbf{F} \& \mathbf{P}$ : Comment as-tu réussi à concilier vie familiale et vie professionnelle?

EPP : J'ai trois enfants et j'ai dû changer de thématique dans les années 80 car je ne pouvais quitter Grenoble, mon mari étant en poste dans cette ville. J'ai dû sacrifier des loisirs lorsque mes enfants étaient petits. Je suis très organisée et mon mari a toujours participé aux tâches familiales. J'ai limité les déplacements et n'ai effectué que de courts séjours à l'étranger. Je suggère qu'on développe l'organisation de vidéoconférences, qui peuvent éviter des déplacements fatigants. J'ajoute que j'ai dû me «battre " pour m'imposer dans le milieu de la biologie structurale. Bien qu'il y ait environ 50\% de femmes à l'IBS, il n'y a aujourd'hui que trois femmes sur dix-huit responsables de groupe.

F\&P : Quels conseils donnerais-tu aux jeunes filles? EPP : Je leur recommanderais d'être très persévérantes. La période la plus difficile pour une mère de famille est quand ses enfants sont très jeunes. Mais cette période est limitée. Mener de front une carrière et des responsabilités familiales permet d'avoir une vie riche et équilibrée. 